DOI: $10.17148 /$ IARJSET.2021.8804

\title{
Sentiment Analysis on Social Media during COVID-19
}

\author{
O Sirisha $^{1}$, C Jyothi Sree ${ }^{2}$, P Jahnavi ${ }^{3}$ \\ Assistant Professor, Computer Science, RBVRR Women's college, Narayanguda, Hyderabad ${ }^{1}$ \\ Assistant Professor, Computer Science, RBVRR Women's college, Narayanguda, Hyderabad ${ }^{2}$ \\ UG - B.com- Business Analytics, RBVRR Women's college, Narayanguda, Hyderabad ${ }^{3}$
}

\begin{abstract}
Sentiment analysis is the technology under Natural Language Processing and text analysis techniques to identify and extract subjective information from text. Sentiment analysis is used in many applications for recommendation and feedback analysis. Sentiment analysis over Social Media offers organizations and individuals a fast and effective way to monitor the publics' feelings towards them and their competitors. There are several challenges facing the sentiment analysis and evaluation process. These challenges become obstacles in analyzing the accurate meaning of sentiments and detecting the suitable orientation of the expressed sentiment. Sentiment analysis is process of extracting information from the user's opinions. Every person shares his or her information on social network sites, blogs, product review websites. This paper focuses on evaluating the predictions of sentiment classifiers, additional feature extractions with practical results on defining the sentiment analysis of COVID-19 using twitter data. The analyses are based on the machine learning algorithms. This article provides an analysis on how people react to a pandemic outbreak, how much they are aware of the disease and its symptoms, what precautionary measures they are taking. Algorithms for sentiment analysis and the steps involved in it. A brief description of complex sequence-based Neural Network sentiment classifiers.
\end{abstract}

Keywords: Sentiment Analysis, Machine Learning, Social Media- Twitter.

\section{INTRODUCTION}

Nowadays, the Internet is becoming worldwide popular, and it is serving as a cost-effective platform for information carriers of social media. Several social media platforms like blogs, posts, tweets are being processed for extracting the people's opinions about a particular situation. By using sentiment analysis, we can interpret the sentiments or emotions of people using some social media sites like twitter. In this article we will analyze various types of "Tweets" gathered during pandemic times. Therefore, our main focus is to do the sentiment analysis on Social Media during COVID-19 to draw some conclusions on people's opinion. Coronavirus disease (COVID-19) first was identified in December 2019 in Wuhan, China and has spread throughout the world. The world has witnessed many pandemic periods, but this pandemic today arouses severe economic problems on a country-scale and world-scale.

Diverse use of social networking sites, like Twitter, speeds up the process of sharing information and having views on and health crises. COVID-19 has been one of Twitter's trending areas throughout January 2020 and it has continued to be debated so far.

Twitter data is useful in exposing public debates and feelings about exciting issues and real knowledge of emerging pandemics. In the ongoing COVID-19 pandemic, several government agencies around the world use Twitter as one of the key means of contact to frequently exchange policy updates and news related to COVID-19 with the general public [6]. Increasing numbers of studies have been collected from Twitter data since the COVID-19 outbreak to understand the general public's reactions and conversations related to COVID- 19 .

\subsection{Sentiment Analysis}

Sentiment analysis, also called opinion mining, is the field of study that analyzes people's opinions, sentiments, appraisals, attitudes, and emotions toward entities and their attributes expressed in written text. The entities can be products, services, organizations, individuals, events, issues, or topics. The field represents a large problem space. Many related names and slightly different tasks, for example, sentiment analysis, opinion mining, opinion analysis, opinion extraction, sentiment mining, subjectivity analysis, affect analysis, emotion analysis, and review mining, are now all under the umbrella of sentiment analysis. The term sentiment analysis perhaps first appeared in Nasukawa and Yi (2003), and the term opinion mining first appeared in Dave et al. (2003). However, research on sentiment and opinion began earlier (Wiebe, 2000; Das and Chen, 2001; Tong, 2001; Morinaga et al., 2002; Pang et al., 2002; Turney, 2002).

\subsection{Twitter}

Twitter is a 'micro blogging' system that allows you to send and receive short posts called tweets. Twitter has become increasingly popular with academics as well as students, policymakers, politicians and the general public. One of the best 


\section{International Advanced Research Journal in Science, Engineering and Technology}

Vol. 8, Issue 8, August 2021

DOI: $10.17148 /$ IARJSET.2021.8804

advantages of Twitter is the ability to connect and share with other users in the Twitter network. Individuals and businesses can share links, photos, and video with their followers and engage in conversations with them.

\section{Problem Definition}

This research aims to capture, process and evaluate people's feelings within a certain timeframe based on the tweets posted on twitter. The study would therefore concentrate on the following things: i. Collect the tweets through Twitter API using the R Shiny package in R programming was used. The Hash tag used for collecting the tweet were \#covid, \#COVID, \#CORONAVIRUS, \#COVIDVACCINE ii. Pre-process the tweets by data cleaning (removing white spaces, links, punctuations, stop words, tokenization, retweet). iii. Calculate the sentiment using the Shiny package and analyze the result. The tweets posted in English have been considered for a sentiment analysis to understand how people from different infected countries have responded during this pandemic situation to cope with it. The collected tweets will be used, pre-processed and applied with text mining algorithms for performing the sentiment analysis.

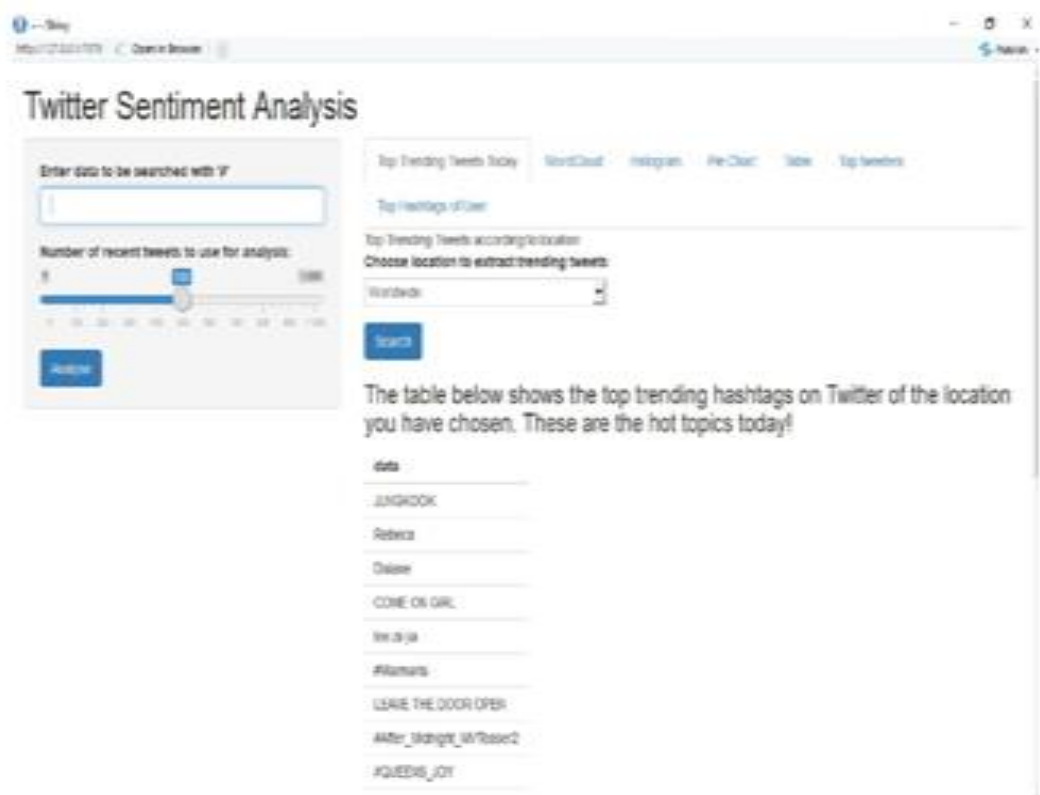

Fig 1.Twitter Sentiment Analysis

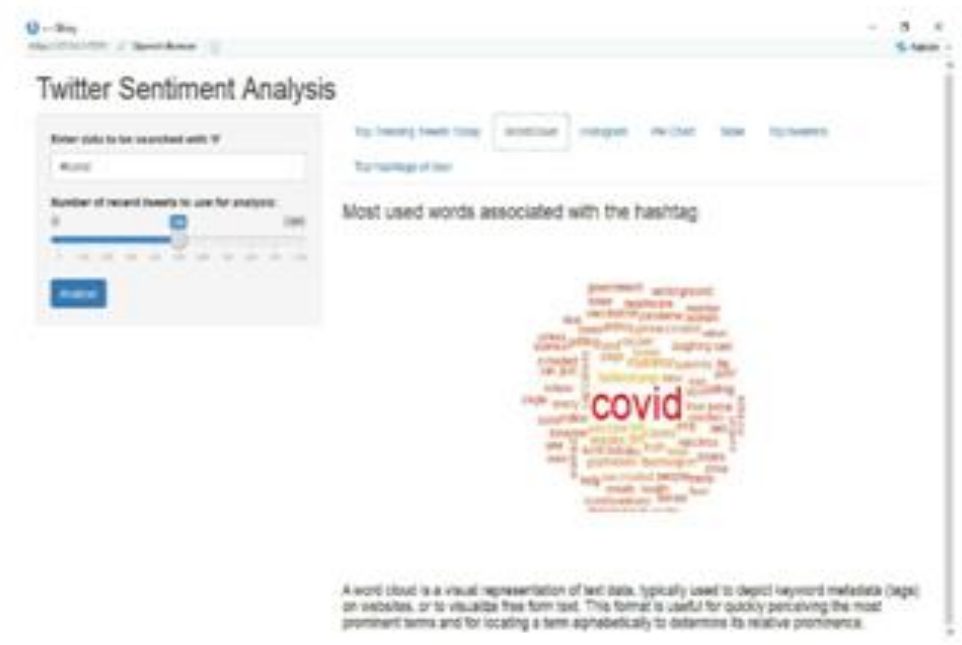

Fig.2.Word Cloud for \#covid

\section{RESEARCH METHOD}

Between $28^{\text {th }}$ June 2021 and 29th July 2021 we created a list of hash tags associated to COVID-19 to check for appropriate tweets. We retrieved the tweets using the advanced programming interface (API) of Twitter's search 


\section{International Advanced Research Journal in Science, Engineering and Technology}

Vol. 8, Issue 8, August 2021

\section{DOI: $10.17148 /$ IARJSET.2021.8804}

application Shiny, which is a web application framework for R that enables to build interactive web applications. Shiny apps are useful to communicate information as interactive data explorations instead of static documents. We carried out a sentiment analysis using tweet text to classify the emotional valence (positive, negative or neutral) of each tweet and prevailing emotions (anger, disgust, fear, happiness, sadness, or surprise).

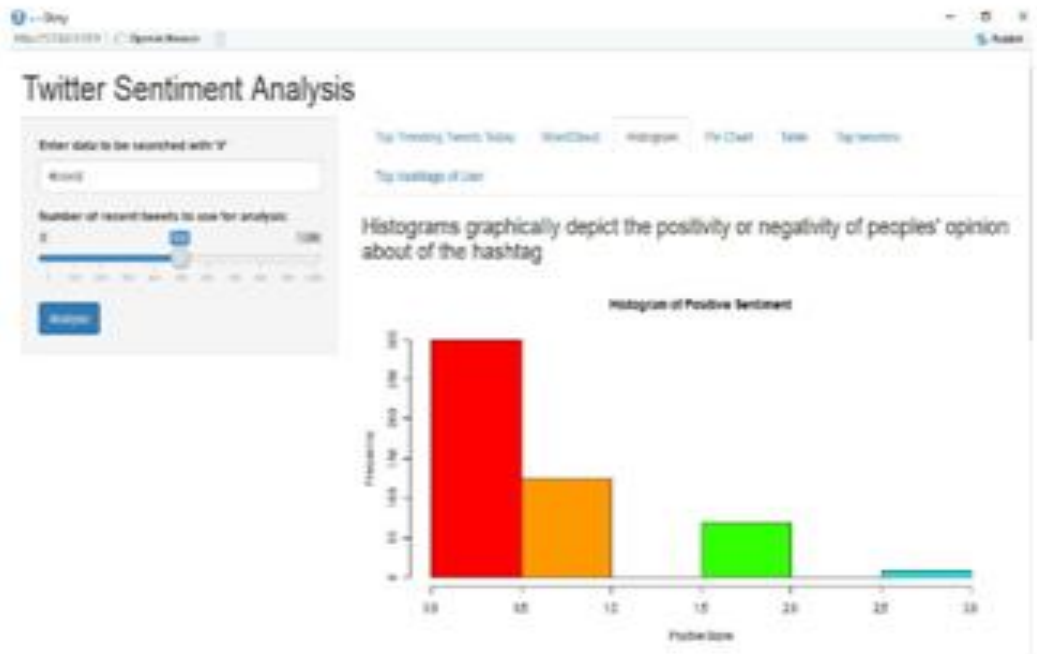

Fig.3.Histogram of Positive Sentiment

Twitter sentiment analysis is done to determine, from tweets, whether people are talking positively or negatively about the topic. Words in the tweet are assigned positive/ negative scores based on their occurrence in the list of words indicating positive/negative sentiment. If positive score is higher tweet is considered to show a positive sentiment, otherwise, negative.

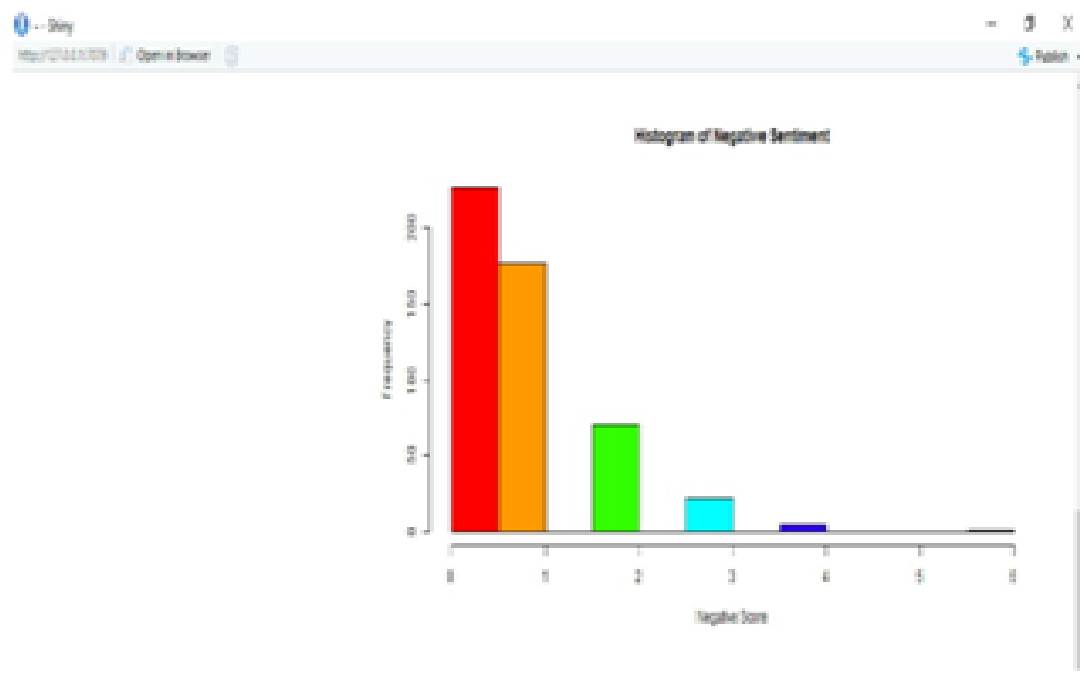

Fig.4.Histogram of Negative Sentiment

A histogram is a graphical representation that organizes a group of data points into user-specified ranges. Similar in appearance to a bar graph, the histogram condenses a data series into an easily interpreted visual by taking many data points and grouping them into logical ranges. 
International Advanced Research Journal in Science, Engineering and Technology

Vol. 8, Issue 8, August 2021

DOI: $10.17148 /$ IARJSET.2021.8804

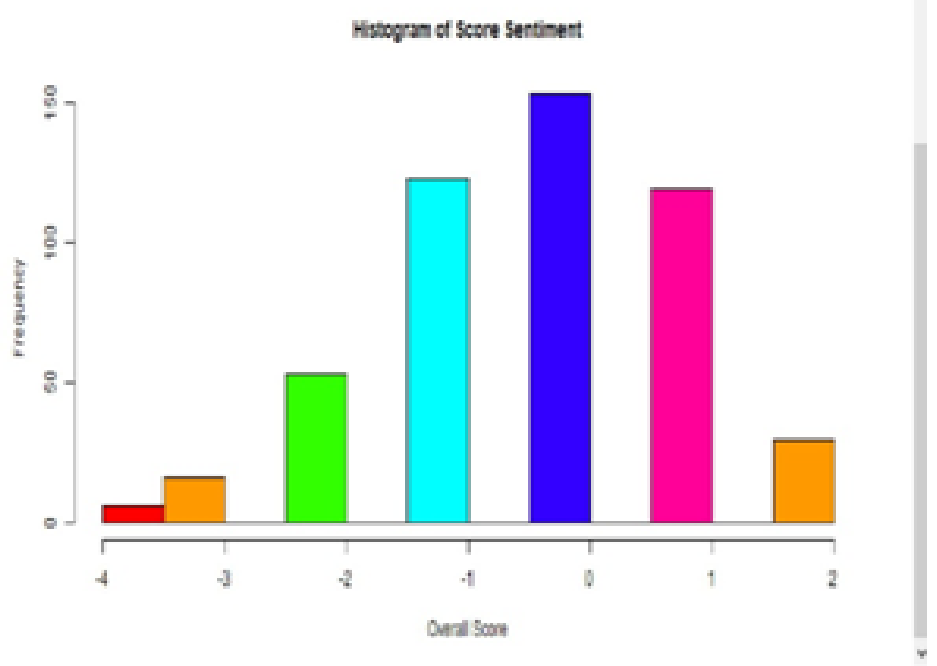

Fig.5.Histogram of Score Sentiment

The histogram of positive sentiment, negative sentiment and Score sentiment of \#covid are shown in the above figures using which we can analyze the people's reaction to COVID-19 pandemic.

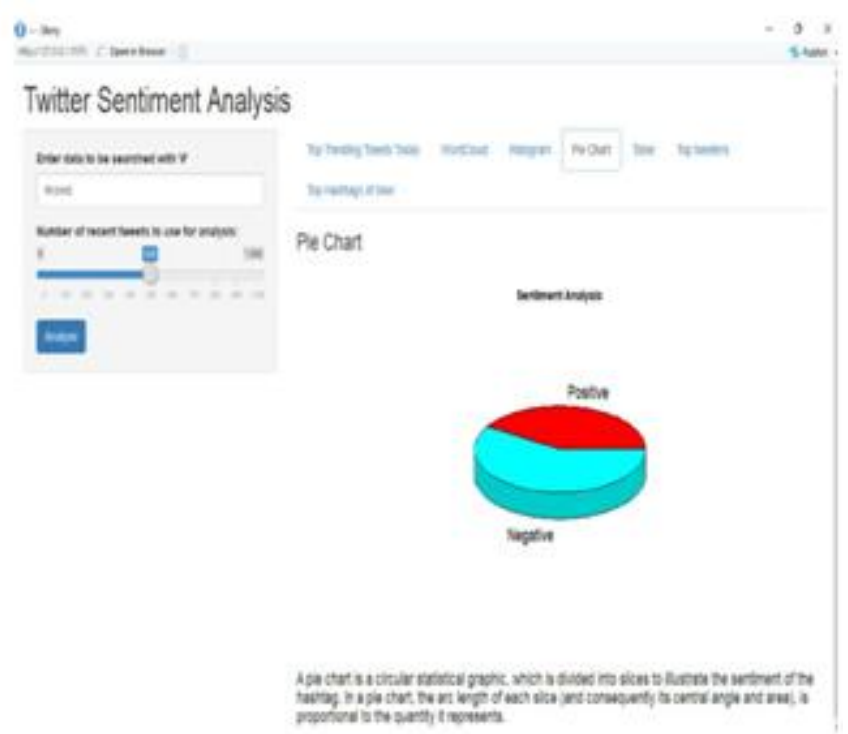

Chart.1.Pie Chart for Sentiment Analysis 


\section{International Advanced Research Journal in Science, Engineering and Technology}

Vol. 8, Issue 8, August 2021

DOI: $10.17148 /$ IARJSET.2021.8804

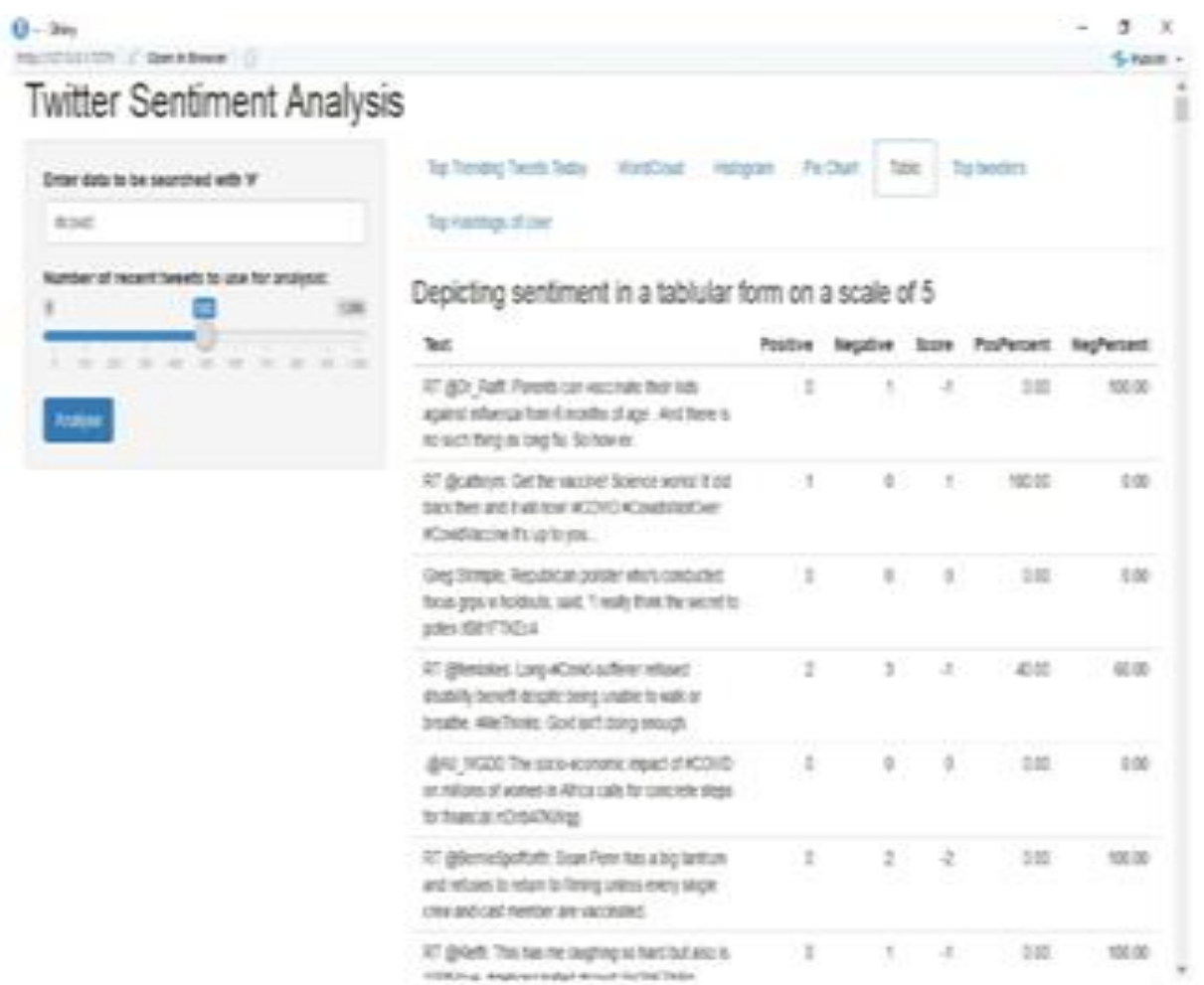

Table.1.Depicting sentiment in a tabular form on scale of 5

\section{CONCLUSION}

This research work aimed at analyzing the sentiments and emotions of the people during the pandemic COVID-19 have been successfully conducted. To maintain the credibility of data and also the ease of extracting tweets of users, the Twitter platform has been chosen for the study.

During the study, it was observed that almost all countries were tweeting about COVID19 with a positive sentiments, since all those people become habitual to COVID-19 and also negative sentiments with equal rates. Similarly, while analyzing the word clouds of different countries, it was concluded that people are tweeting words like Pandemic, COVID, Virus, Hospitals, Health, Fight, Stay, Safe, Help, Emergency, Death and Masks with different emotions.

This study provided a good analysis on sentiments and mind sets of people on Covid-19 and enabled us to understand that almost the same level of thinking of people all over the world. This research can be used for future works to examine the shifting emotions and feelings of individuals from these nations and to check if there are noticeable changes over time in them.

\section{REFERENCES}

Public_Sentiment_Analysis_on_Twitter_Data

Sentiment Analysis on the impact of corona virus in social life using the BERT model

https://www.worldometers.info/coronavirus/, accessed on July 28, 2021.

4. A.Pak and P. Paroubek. „Twitter as a Corpus for Sentiment Analysis and Opinion Mining". In Proceedings of the Seventh Conference on International Language Resources and Evaluation, 2010, pp.1320-1326.

5. Dmitry Davidov, Ari Rappoport." Enhanced Sentiment Learning Using Twitter Hashtags and Smileys". Coling 2010: Poster Volumepages $241\{249$, Beijing, August 2010

6. Neethu M,S and Rajashree R," Sentiment Analysis in Twitter using Machine Learning Techniques" 4th ICCCNT 2013, at Tiruchengode, India. IEEE - 31661 